\title{
Métodos ativos de aprendizagem como inovação na educação em enfermagem
}

\author{
Active learning methods as an innovation in nursing education
}

\author{
Maria Aline Moreira Ximenes' • Maria Girlane Sousa Albuquerque Brandão² • Joselany Áfio Caetano ${ }^{3}$ \\ Lívia Moreira Barros ${ }^{4}$
}

\begin{abstract}
RESUMO
Objetivo: descrever o uso de métodos ativos de aprendizagem em encontros de monitoria do módulo curricular de Saúde do Adulto no curso de enfermagem. Método:Trata-se de estudo descritivo, qualitativo, realizado em Instituição de Ensino Superior Pública, de janeiro a maio de 2018. O módulo possuía 30 acadêmicos matriculados. Ao total, foram realizados 10 encontros de monitoria, que tiveram duração média de 90 minutos. Em todos os encontros de monitoria foram empregadas metodologias ativas. Resultados: Por meio de metodologias ativas de aprendizagem baseada em problemas, simulação com caso clínico, construção de procedimento operacional padrão, resolução de questões, roda científica e Jogo de Tabuleiro, houve uma relação direta de troca de conhecimentos e experiências entre acadêmicos e monitores. Em todos os encontros observou-se participação assídua dos acadêmicos, pontualidade, bons resultados nas atividades realizadas. Conclusão: As metodologias ativas utilizadas no ensino-aprendizagem no curso de Enfermagem possibilitaram aos acadêmicos e monitores - desenvolvimento de habilidades criativas e diferentes formas de ministrar temas relevantes na formação em saúde, o que fortalece o desenvolvimento de um olhar crítico-reflexivo dos discentes e aponta caminhos para a gênese de uma nova estruturação curricular, moldada nas estratégias horizontais de ensino.
\end{abstract}

Palavras-chave: Ensino Superior, Monitoria, Inovação

\begin{abstract}
Objective: describe the use of active methodologies in monitoring meetings of the Adult Health curriculum module in the nursing course. Method: It is descriptive, qualitative study, carried out at a Public Higher Education Institution in Northeast Brazil, from January to May 2018. The module had 30 students enrolled. In total, 10 monitoring meetings were held, which lasted an average of 90 minutes. Active monitoring methodologies were used in all monitoring meetings. Results:Through active methodologies of problem-based learning, simulation with clinical case, construction of standard operating procedure, resolution of questions, scientific round and Board Game, there was a direct relationship of exchange of knowledge and experiences between academics and monitors. In all meetings, there was constant participation of academics, punctuality, good results in the activities carried out. Conclusion:The active methodologies used in teaching-learning in the Nursing course allowed academics and monitors to develop creative skills and different ways of teaching relevant topics in health education. Keywords: Colleges of Education, Tutoring, Innovation.
\end{abstract}

I Enfermeira. Mestranda em Enfermagem pela Universidade Federal do Ceará (UFC). Departamento de Enfermagem. Fortaleza, Ceará, Brasil. E-mail: aline.ximenesII@hotmail.com Orcid: https://orcid.org/0000-0002-I674-3357

2 Enfermeira. Mestranda em Enfermagem pela Universidade da Integração Internacional da Lusofonia Afro-Brasileira (UNILAB). Departamento de Enfermagem. Redenção,

Ceará, Brasil. E-mail: girlane.albuquerque@yahoo.com.br Orcid: http://orcid.org/0000-0002-9925-4750

3 Doutora em Enfermagem. Docente da Universidade Federal do Ceará (UFC). Departamento de Enfermagem. Fortaleza, Ceará, Brasil. E-mail: joselany@ufc.br Orcid: http://orcid.org/0000-0002-0807-056X

4 Doutora em Enfermagem. Docente da Universidade da Integração Internacional da Lusofonia Afro-Brasileira (UNILAB). Departamento de Enfermagem. Redenção, CE, Brasil. E-mail: livia.moreirab@hotmail.com Orcid: http://orcid.org/0000-0002-0I74-2255 


\section{INTRODUÇÃO}

As instituições superiores de ensino são responsáveis pela formação de profissionais qualificados e capacitados para atuar com competência e habilidade. Nos cursos da área da saúde, é fundamental que Universidade e corpo docente elaborem estratégias pautadas na formação de acadêmicos como um ser ético, histórico, crítico, reflexivo, transformador, com visão indissolúvel entre teoria e prática humanizada ${ }^{(1)}$.

No contexto dessa formação, ganha destaque a monitoria acadêmica, ferramenta de apoio pedagógico entre discente-monitor e discente-acadêmico, pois ambos têm oportunidade de aprofundar conhecimentos, fortalecer habilidades teórico-práticas e esclarecer dúvidas, reduzindo fragilidades inerentes a uma área de conhecimento(2).

Considera-se a monitoria acadêmica como programa de incentivo à educação por contribuir com o avanço do conhecimento e propiciar a possibilidade de nivelamento, uma vez que o monitor atende às demandas dos alunos nos anos anteriores. Além disso, proporciona ao monitor estímulos para aperfeiçoar habilidades técnicas, relações interpessoais e liderança. Consequentemente, torna-se uma oportunidade para o discente construir novas práticas, levando em consideração suas próprias deficiências e necessidades enquanto aluno.

Pesquisa experimental realizada na Espanha evidenciou melhoria estatisticamente significante nos escores de informação e compreensão no grupo intervenção, que recebeu nove momentos de monitoria acadêmica, quando comparadas ao grupo controle. Além do mais, a maioria dos alunos afirmou que os encontros os auxiliaram a lidar melhor com as dificuldades educacionais e emocionais da disciplina e se concentrar em técnicas de estudo ${ }^{(3)}$.

Associado as práticas de monitoria, destaca-se a utilização de metodologias ativas (MA) como estratégias para desenvolvimento e fortalecimento de competências durante o processo de ensino aprendizagem. $O$ uso dessas ferramentas estimula habilidades para a vida profissional do enfermeiro, por propiciar uma aprendizagem contextualizada com visão interdisciplinar do conhecimento.

Além disso, oportuniza uma educação crítico-reflexiva, com base em estímulo no processo de aprendizagem, que resulta em envolvimento por parte do educando na busca pelo conhecimento, as MA podem estimular os monitores a vocação para docência, tendo em vista que promove a cooperação entre os corpos docente e discente, em benefício da qualidade do ensino, ministrado pela Instituição(4).

Ao considerar que o ensino de procedimentos de enfermagem durante atividades de monitoria requer 0 uso de recursos que venham a favorecer o processo de aprendizagem e facilitar a compreensão dos discentes em relação ao conteúdo, as MA podem coadjuvar na ob- tenção de uma visão clínica e tomada de decisão diante de alterações no estado de saúde do paciente adulto.

Dessa forma, o estudo vem a contribuir com a ciência da enfermagem, por relatar experiências exitosas com uso de MA em atividade de monitoria acadêmica, que oportunizaram habilidades docentes e efetividade de novos métodos de ensino-aprendizagem. Logo, o objetivo desse estudo é descrever o uso de MA em encontros de monitoria do módulo curricular de Saúde do Adulto em um curso de enfermagem.

\section{MÉTODOS}

Estudo descritivo de abordagem qualitativa, realizado em Instituição de Ensino Superior (IES) Pública no interior do estado do Ceará, Brasil, no período de janeiro a maio de 2018, durante o módulo acadêmico de Saúde do Adulto.

O módulo Saúde do Adulto integra a grade curricular do Curso de Enfermagem de Universidade Pública Cearense e possui carga horária de 160 horas. A monitoria no referido módulo condensa conteúdos teóricos e práticos específicos do processo saúde-doença clínico e cirúrgico da população adulta, e configura-se como oportunidade de praticar procedimentos, desenvolver habilidades clínicas e estimular o trabalho em equipe necessário durante as vivências práticas.

Os encontros foram realizados no campus de Ciências da Saúde da referida IES, em sala de aula e laboratório de enfermagem. $O$ módulo possuía 30 acadêmicos matriculados. Ao total, foram realizados 10 encontros de monitoria, que tiveram duração média de 90 minutos. Em todos os encontros de monitoria foram ministrados por duas monitoras, em que foram empregadas MA, tais como: aprendizagem baseada em problemas (PBL), simulação com caso clínico, construção de procedimento operacional padrão (POP), resolução de questões, roda científica e Jogo de Tabuleiro.

O planejamento para as atividades de monitoria do módulo realizou-se durante o mês de janeiro de 2018 , mediante orientação da docente coordenadora do módulo e pesquisas na literatura acerca do tema. Elaborouse plano de atividades, o qual foi avaliado e aprovado pela coordenadora do módulo, e, em seguida, apresentado aos acadêmicos durante o primeiro encontro.

Ao final de todas as aulas de monitoria, os aspectos mais relevantes das experiências e as impressões acerca de participação e desenvolvimento dos acadêmicos eram registrados em diário de campo que continha informações sobre presença e participação dos alunos, assim como impressões das monitoras sobre aspectos importantes da experiência. No último encontro, aplicou-se um instrumento de avaliação das monitorias, de autoria própria, que continha questões fechadas tipo likert e abertas 
sobre: os conteúdos apresentados (planejamento e clareza); metodologias utilizadas (aprendizagem, avaliações e tempo); avaliação das monitoras (domínio, pontualidade e disponibilidade) e autoavaliação.

A pesquisa foi aprovada pelo Comitê de Ética em Pesquisa (CEP) da Universidade Estadual Vale do Acaraú, sob parecer de $n^{\circ} 2.260 .807 / 2017$, seguiu as recomendações da Resolução n. ${ }^{\circ}$ 466/12, do Conselho Nacional de Saúde.

\section{RESULTADOS}

As metodologias ativas elegidas neste estudo tratamse de experiências exitosas de outros trabalhos acadêmicos com práticas de monitoria, sugestões acadêmicas e pesquisas na literatura. Em todos os encontros observou-se participação assídua dos acadêmicos, pontualidade, bons resultados nas atividades realizadas em todos os encontros. -

QUADRO 1 - Descrição das atividades de monitoria. Sobral, Ceará, Brasil. 2018.

\begin{tabular}{|c|c|c|c|}
\hline Metodologia Ativa & $\begin{array}{l}\text { Temática do } \\
\text { Encontro }\end{array}$ & Objetivo & Descrição das atividades \\
\hline \multirow[t]{2}{*}{$\begin{array}{l}\text { Simulação com caso } \\
\text { clínico }\end{array}$} & $\begin{array}{l}\text { Sistematização } \\
\text { da Assistência de } \\
\text { Enfermagem } \\
\text { (SAE) }\end{array}$ & $\begin{array}{l}\text { Realizar plano de } \\
\text { cuidados com base } \\
\text { na SAE }\end{array}$ & $\begin{array}{l}\text { A sala de aula simulou um consultório para a consulta de } \\
\text { enfermagem. As monitoras apresentaram o caso clínico e os } \\
\text { discentes foram orientados a construir o histórico do paciente. } \\
\text { Após a coleta das informações, foi estabelecido tempo para que } \\
\text { os grupos pudessem identificar diagnósticos de enfermagem } \\
\text { do caso, resultados esperados e intervenções. Em seguida os } \\
\text { grupos encenaram para toda a turma o desfecho da consulta } \\
\text { com orientações/intervenções de enfermagem ao paciente. }\end{array}$ \\
\hline & $\begin{array}{l}\text { Preparo e } \\
\text { administração de } \\
\text { medicamentos por } \\
\text { via endovenosa }\end{array}$ & $\begin{array}{l}\text { Praticar o preparo } \\
\text { e administração de } \\
\text { medicamentos por via } \\
\text { endovenosa }\end{array}$ & $\begin{array}{l}\text { Os acadêmicos foram divididos em pares, de forma que } \\
\text { pudessem ser melhor supervisionados pelas monitoras. Cada } \\
\text { aluno realizava o procedimento de preparo da medicação e em } \\
\text { seguida, a punção venosa, nos manequins do laboratório. }\end{array}$ \\
\hline \multirow[t]{2}{*}{$\begin{array}{l}\text { Aprendizagem } \\
\text { Baseada em } \\
\text { Problemas }\end{array}$} & $\begin{array}{l}\text { Higiene e conforto } \\
\text { do paciente do leito }\end{array}$ & $\begin{array}{l}\text { Discutir questões } \\
\text { sobre higiene } \\
\text { e conforto do } \\
\text { paciente durante a } \\
\text { internação e realizar } \\
\text { o procedimento de } \\
\text { banho no leito }\end{array}$ & $\begin{array}{l}\text { Elaborou-se situação clínica com paciente em cuidados } \\
\text { paliativos, em que os discentes deveriam identificar diagnósticos, } \\
\text { condutas, restrições do paciente e avaliação crítica reflexiva dos } \\
\text { cuidados de higiene. Em grupos, houve a discussão do caso e } \\
\text { posteriormente com a turma, onde cada equipe socializou sua } \\
\text { interpretação do caso. Por fim, ocorreu a prática de banho no } \\
\text { leito realizada pelas equipes em manequins sob a supervisão } \\
\text { das monitoras. }\end{array}$ \\
\hline & Manejo de Feridas & $\begin{array}{l}\text { Desenvolver avaliação } \\
\text { clínica, construção de } \\
\text { planos de cuidados } \\
\text { em pacientes com } \\
\text { feridas e realizar o } \\
\text { curativo. }\end{array}$ & $\begin{array}{l}\text { Foi construído caso clínico com feridas. Cada equipe recebeu } \\
\text { o caso com perguntas chaves, as quais seriam respondidas, e } \\
\text { em seguida discutidas entre a turma. Cada equipe identificou } \\
\text { problemas sistêmicos relacionados, tipo de ferida, grau de } \\
\text { contaminação e realizou o curativo ideal para o paciente } \\
\text { hipotético em manequim. }\end{array}$ \\
\hline \multirow[b]{2}{*}{ Roda Científica } & Oxigenoterapia & $\begin{array}{l}\text { Discutir sobre } \\
\text { indicações e formas } \\
\text { de administração de } \\
\text { oxigênio suplementar }\end{array}$ & $\begin{array}{l}\text { Foram escolhidos artigos nacionais publicados nos últimos dois } \\
\text { anos sobre oxigenoterapia. A turma foi dividida em duplas, cada } \\
\text { dupla recebeu um artigo, o qual deveria ser lido e em seguida } \\
\text { explicado para a turma. Durante a apresentação das temáticas } \\
\text { presentes no artigo, as monitoras realizaram perguntas-chaves } \\
\text { sobre os textos, as quais foram discutidas em plenária. }\end{array}$ \\
\hline & $\begin{array}{l}\text { Hemotransfusão } \\
\text { e assistência de } \\
\text { enfermagem }\end{array}$ & $\begin{array}{l}\text { Discutir sobre } \\
\text { questões sobre } \\
\text { Hemotransfusão e o } \\
\text { Papel da enfermagem } \\
\text { nesse processo }\end{array}$ & $\begin{array}{l}\text { Foram escolhidos artigos nacionais publicados nos últimos dois } \\
\text { anos sobre Hemotransfusão e assistência de enfermagem. A } \\
\text { turma foi dividida em duplas, cada uma recebeu um artigo, o qual } \\
\text { deveria ser lido em seguida explicado para a turma. Durante a } \\
\text { apresentação das temáticas presentes nos artigos, as monitoras } \\
\text { realizaram perguntas-chaves sobre os textos, as quais foram } \\
\text { discutidas por toda a turma. }\end{array}$ \\
\hline $\begin{array}{l}\text { Resolução de } \\
\text { questões em grupo }\end{array}$ & $\begin{array}{l}\text { Enfermagem } \\
\text { Cirúrgica }\end{array}$ & $\begin{array}{l}\text { Praticar } \\
\text { conhecimentos } \\
\text { resolvendo questões } \\
\text { sobre Assistência } \\
\text { de Enfermagem em } \\
\text { Centro Cirúrgico }\end{array}$ & $\begin{array}{l}\text { Para esse encontro, foram selecionadas questões de concurso } \\
\text { com base na temática Enfermagem Cirúrgica, foram elaborados } \\
\text { slides explicitando a resolução das questões, utilizando recursos } \\
\text { de imagem e vídeo. Inicialmente a questão era projetada, e um } \\
\text { aluno era sorteado a responder para toda a turma, em seguida } \\
\text { a questão era explicada e discutida para toda a sala pelas } \\
\text { monitoras. }\end{array}$ \\
\hline
\end{tabular}




\begin{tabular}{|c|c|c|c|}
\hline \multirow{2}{*}{$\begin{array}{l}\text { Construção de } \\
\text { Procedimento } \\
\text { Operacional Padrão } \\
\text { (POP) }\end{array}$} & Distúrbios urinários & $\begin{array}{l}\text { Sistematização } \\
\text { e prática do } \\
\text { procedimento de } \\
\text { sondagem vesical. }\end{array}$ & $\begin{array}{l}\text { A turma foi dividida em quatro equipes, e cada equipe construiu } \\
\text { dois POP do procedimento, um voltado para o homem e outro } \\
\text { para a mulher. Após esse processo, houve prática no manequim } \\
\text { de forma que as demais equipes pudessem assistir e discutir } \\
\text { os erros observados, os pontos importantes e dicas para a } \\
\text { realização do procedimento. }\end{array}$ \\
\hline & $\begin{array}{l}\text { Distúrbios } \\
\text { gastrointestinais }\end{array}$ & $\begin{array}{l}\text { Sistematização } \\
\text { e prática do } \\
\text { procedimento } \\
\text { de sondagem } \\
\text { gastrointestinal }\end{array}$ & $\begin{array}{l}\text { Em quatro, construiu-se POP para prática sondagem } \\
\text { nasogástrica, orogástrica, nasoenteral e oroenteral. Após } \\
\text { a construção, houve demonstração dos procedimentos em } \\
\text { manequim. }\end{array}$ \\
\hline Jogo de Tabuleiro & $\begin{array}{l}\text { Revisão de } \\
\text { conteúdos }\end{array}$ & $\begin{array}{l}\text { Revisar todas as } \\
\text { temáticas trabalhadas } \\
\text { durante a monitoria }\end{array}$ & $\begin{array}{l}\text { Revisão dos conteúdos anteriores, onde a turma foi dividida } \\
\text { em cinco grupos e cada um possuía um representante. Foram } \\
\text { elaboradas } 50 \text { questões teóricas e práticas sobre todos os } \\
\text { assuntos abordados na monitoria, a cada pergunta feita, a equipe } \\
\text { tinha um minuto para responder ou realizar o procedimento. O } \\
\text { jogo possuía } 25 \text { casas, incluindo avançar, voltar, trocar com o } \\
\text { colega e responder duas perguntas. O dado era lançado pelo } \\
\text { representante da equipe e o número apresentado correspondia } \\
\text { à quantidade de casas avançadas, mas para isso acontecer, } \\
\text { deveriam responder corretamente a pergunta, caso contrário } \\
\text { voltava ao início do jogo. }\end{array}$ \\
\hline
\end{tabular}

Fonte: Própria do Autor, 2018.

\section{DISCUSSÃO}

Em todas as atividades houveram trocas de conhecimentos e experiências entre docentes e monitores, com vantagens no processo de ensino-aprendizagem acadêmico. A literatura infere que enquanto os alunos monitores revisam os conteúdos teóricos, essenciais para as práticas de Enfermagem, exercitam a habilidade de orientar condutas e instruções, além de alicerçar sua formação profissional e liderança ${ }^{(5)}$. Além disso, a utilização de MA de ensino-aprendizagem em atividades de monitoria acadêmica é um fator que potencializa o desenvolvimento de habilidades comunicativas e clínicas nos estudantes.

Conforme demonstrado no Quadro I, para as temáticas de Consulta de Enfermagem e Preparo e administração de medicamentos por via endovenosa, utilizou-se como metodologia ativa a simulação com caso clínico. A metodologia de simulação com caso clínico propiciou um espaço de raciocínio diagnóstico em enfermagem, com apresentação de intervenções mediante a avaliação do histórico do paciente simulado.

A estruturação de metodologias de simulação clínica permite o aprendizado do diagnóstico de enfermagem, favorável para a construção de um raciocínio clínico, crítico e acurado. Estudo realizado com acadêmicos de enfermagem de Universidade em São Paulo identificou que a realização de cenários simulados com caso clínico oferece experiências cognitivas e psicomotoras, que contribuem para a transferência de conhecimento da sala de aula/laboratório de enfermagem para os ambientes clínicos realísticos ${ }^{(6)}$.

Tal resultado é favorável ao progresso profissional dos acadêmicos, uma vez que, somente com conhecimento e raciocínio clínico é possível instituir os diagnósticos de enfermagem e implementar as intervenções necessárias frente ao estado de saúde do paciente.

A Prática de procedimento de Terapia intravenosa teve como objetivo a execução do preparo de medicação e realização de punção venosa nos manequins. Nesse quesito vale inferir, que a punção venosa é procedimento comum nos cuidados de saúde, realizada com intuito de infundir líquidos no lúmen intravascular e deve ser alvo recorrente na educação em enfermagem, ministrada de forma pedagógica, que garanta o aprendizado do futuro enfermeiro(7).

Ressalta-se que durante as vivências práticas hospitalares, geralmente, a técnica de punção venosa é o primeiro procedimento que os acadêmicos têm oportunidade de realizar. A execução de punção venosa é uma técnica simples da rotina hospitalar, no entanto, sua prática merece destaque, em face do risco de infecções de corrente sanguínea e autocontaminação quando há realização incorreta do procedimento. Portanto, é fundamental que os acadêmicos recebam as instruções e treinamentos necessários para evitar falhas e garantir a biossegurança.

A metodologia de PBL foi utilizada como estratégia de aprendizagem para as temáticas de higiene e conforto do paciente do leito e manejo do paciente com feridas, em que os acadêmicos exercitaram suas habilidades de diagnósticos de enfermagem, condutas, restrições do paciente e avaliação crítica.

Pesquisa com acadêmicos de enfermagem e psicologia de Universidade Pública Cearense identificou que essa metodologia foi aprovada pelos estudantes, que se autoavaliaram como atuantes e reflexivos na construção do crescimento pessoal e profissional, além da horizontalida- 
de do método, que proporciona espaços de discussões e desenvolvimento de habilidades, como a fala e a escuta(8).

Isso reforça a importância de substituir os métodos tradicionais de ensino e oportunizar ao discente a participação em atividades que estimulem o pensamento crítico, atuação em equipe, reflexão sobre sua formação e atuação profissional. A resolução de situações-problema por meio do PBL poderá contribuir na gênese de profissionais mais ativos, autônomos e dialógicos.

Outra MA utilizada foi a Roda Científica, que contemplou as temáticas de Oxigenoterapia e assistência de enfermagem na Hemotransfusão. Ler e interpretar artigos científicos é uma prática necessária e de extrema relevância no campo da enfermagem. Contudo, a leitura de artigos científicos exige domínio de habilidades específicas, como a capacidade de identificar a organização textual, os conceitos básicos do tema/área de conhecimento tratado no texto e familiarização mínima da linguagem científica ${ }^{(9)}$.

Os artigos são os principais divulgadores de informações e pesquisas no meio acadêmico. Assim, enfatiza-se a relevância de uma familiarização dos alunos universitários com a leitura de artigos científicos para aquisição de novos conhecimentos.

A metodologia de resolução de questões em grupo foi eleita para debater questões de concursos de enfermagem sobre a temática de Enfermagem Cirúrgica. A resolução de questões em grupo é uma estratégia que pode ser utilizada para consolidar e fomentar a aprendizagem. Essa estratégia pode promover uma melhora na forma como o estudante organiza a informação e possibilita a retenção de conteúdos por um tempo maior ${ }^{(10)}$.

$O$ estudo baseado na resolução de questões pode fornecer a identificação de temas e pontos que demandam um estudo mais aprofundado, o que solicita uma autoavaliação para nortear futuras sessões de estudo. Dessa forma, ressalta-se a relevância de estimular o aluno durante a graduação em enfermagem à resolução de questões, uma vez que, em muitas unidades de saúde, a forma de ingresso para atuação profissional trata-se de concursos, em que o enfermeiro que pleiteia uma vaga no mercado de trabalho deve estar apto a resolução de questões de forma hábil e ágil.

A Construção de Procedimento Operacional Padrão (POP) foi empregada na assistência de enfermagem nos distúrbios urinários e gastrintestinais.A construção e utilização do POPs é uma ferramenta que pode ser utilizada para melhorar a qualidade da assistência prestada e padronizar as intervenções de enfermagem, pois viabiliza a segurança dos procedimentos, como cateterismos gástricos e vesicais, embasados em evidências científicas, 0 que fortalece a confiabilidade da assistência prestada ${ }^{(1,12)}$.

É função privativa do enfermeiro os cuidados e orientações necessárias para a realização de cateterismo gástrico e vesical.Assim, os acadêmicos devem receber capacitação para realizar o procedimento de maneira correta com as devidas precauções de risco de infecção.

O jogo de tabuleiro foi utilizado como metodologia de resgate e revisão dos conteúdos elencados nos encontros anteriores. Estudos apontam que o jogo de tabuleiro é uma tecnologia leve-dura que se configura como ferramenta lúdica capaz de estimular a assimilação e resgate de conhecimentos em conteúdo de enfermagem $^{(13,14)}$. Percebe-se, assim, a importância da utilização de MA, como os jogos que favorecem um ambiente de aprendizagem por meio da interação dos acadêmicos, em práticas recreativas como instrumento educacional e desenvolvimento do raciocínio lógico.

As MA utilizadas na monitoria do módulo de Saúde do Adulto são pontos de partida para o avanço no processo de ensino-aprendizagem, com discussão de casos clínicos, reflexão, integração cognitiva e reelaboração de novas práticas. Os resultados positivos da monitoria são ampliados para os pacientes, que receberão cuidados por estudantes com maior preparo para desenvolver os diagnósticos de enfermagem, intervenções e procedimentos pautados na integralidade do cuidado, valores éticos e humanos ${ }^{(1)}$.

Com base na avaliação dos discentes constatou-se que as MA no ensino-aprendizagem no curso de Enfermagem possibilitaram aos acadêmicos o desenvolvimento de novas habilidades confiança para realização de procedimentos relevantes para formação em saúde. Assim como propiciou o desenvolvimento de um olhar crítico-reflexivo sobre uma estruturação curricular, moldada nas estratégias horizontais de ensino.

As estratégias de ensino aprendizagem em instituições de ensino superior devem, portanto, buscar romper a lógica do ensino focado na transmissão de conhecimentos de maneira vertical e ir além da metodologia tradicional, buscando usar métodos ativos, com ênfase em situações problema, estudos de casos clínicos, aprendizagem em grupo e aulas participativas com utilização de novas tecnologias para o ensino.

\section{CONCLUSÃO}

A aplicação das MA colaborou com a inovação de práticas docentes e resultados satisfatórios de aprendizagem dos discentes. Logo espera a perpetuação dessa estratégia nas IES, e que outros módulos curriculares, além de Saúde do Adulto, possam apoderar-se das metodologias ativas de aprendizagem. Infere-se a necessidade de ininterrupção da discussão e utilização das MA no ensino superior de enfermagem, com vistas a implementação de uma educação problematizadora e inclusiva, com vistas ao avanço na formação de profissionais em saúde críticos, reflexivos e transformadores da realidade. 


\section{REFERÊNCIAS}

I. Baricati CCA, Martins JT, Yagi MCN, Kreling MCGD, Karino ME, Volpato MP. Monitoria: metodologia ativa na prática do cuidar em um curso de enfermagem. Braz. J. Surg. Clin. Res., [Internet]. 2018 [cited 2019 July 12]; 2I(I):76-78. Available from: https://www.mastereditora.com.br/periodico/2017I204_19045I.pdf

2. Fernandes NC, Cunha RR, Brandão AF, Cunha LL, Barbosa PD, Silva CO, Silva MSA. Academic mentoring and care for a person with a stoma: experience report. Rev Min Enferm., [Internet]. 2015 [cited 2019 Jan 25]; 19(2):242-245. Available from: http://reme.org.br/artigo/detalhes/ 1018

3. Guerra MD, Lima M, Lima JS. Effectiveness of Tutoring to Improve Academic Performance in Nursing Students at the University of Seville. J of New Approaches in Educ Research., [Internet]. 2017 [cited 2019 Jan 20]; 6(2): 93-102.Available from: https://naerjournal.ua.es/article/view/v6n2-2

4. Macedo KDS, Acosta BS, Silva EB, Souza NS, Beck CLC, Silva KKD. Metodologias ativas no ensino em saúde. Escola Anna Nery., [Internet]. 2018 [cited 2019 Jan 20]; 22(3): I-9.Available from: http://www.scielo.br/pdf/ean/v22n3/pt_|4|48|45-ean-22-03-e20।70435.pdf

5. Marcuzzo S, Campos PM, Schneider J, Régis CC. Strategies for motivating embryology learning: an experience report in the nursing course of the federal university of Rio Grande do Sul. Rev. Inter. Educ. Sup., [Internet]. 2019 [cited 2019 Feb 16]; 5(I): I-I4. Available from: https://periodicos.sbu. unicamp.br/ojs/index.php/riesup/article/view/8653470

6. Garbuio DC, Oliveira AR de S, Kameo SY, Melo ES, Dalri MCB, Carvalho EC. Simulação clínica em enfermagem: relato de experiência sobre a construção de um cenário. J Nurs UFPE on line., [Internet]. 2016 [cited 2019 Jan 20]; I0(8): 3|49-55. Available from: http://dx.doi.org//0.5205/ reuol.9373-82I34-I-RVI00820I645

7. González López JL, Arribi Vilela A2, Fernández del Palacio E, Olivares Corral J, Benedicto Martí C, Herrera Portal P. Indwell times, complications and costs of open vs closed safety peripheral intravenous catheters: a randomized study. J Hosp Infect., [Internet]. 2014 [cited 2019 Jan 2I]; 86(2): II7-6. Available from: https://www.ncbi.nlm.nih.gov/ pubmed/24373830
8. Sampaio CL, Neri MFS, Araújo MAM, Caetano JA, Eloia SMC, Souza AMA. Problem-Based Learning in Teaching of Thanatology in Undergraduate Nursing Program. Escola Anna Nery., [Internet]. 2018 [cited 2019 Jan 16]; 22(3): I-7.Available from: http://www.scielo.br/pdf/ean/v22n3//4I4-8I45ean-22-03-e20180068.pdf

9. Silveira MIM, Ritti-Dias FG. A prática de leitura entre alunos graduandos: foco no artigo científico. Rev X., 20I3; I(I): 28-37.

10. Dunlosky J, Rawson KA, Marsh EJ, Nathan MJ, Willingham DT. Improving Students' Learning With Effective Learning Techniques: PromisingDirections From Cognitive and Educational Psychology. Psychol Sci Public Interest., [Internet]. 2013 [cited 2019 Jan 16]; I4(I): 4-58. Available from: https://www.ncbi.nlm.nih.gov/pubmed/26173288

II. Pereira LR, Carvalho MF, Santos JS, Machado GAB, Maia MAC, Andrade RD. Avaliação de procedimentos operacionais padrão implantados em um serviço de saúde. Arq. Ciênc. Saúde., [Internet]. 2017 [cited 2019 Jan 21]; 24(4): 47-5I. Available from: http://www.cienciasdasaude.famerp. br/index.php/racs/article/view/840/728

12. Sales CB, Bernardes A, Gabriel CS, Brito MFP, Moura AA, Zanetti ACB. Standard Operational Protocols in professional nursing practice: use, weaknesses and potentialities. Rev Bras Enferm., [Internet]. 2018 [cited 2019 Jan 21]; $71(I): 126-34$. Available from: http://www.scielo.br/pdf/reben/v7In I/pt_0034-7I67-reben-7I-0I-0I26.pdf

13. Amaral MFN, Machado ACC, Santos LNC, Pereira BB. O processo de construção de um jogo sobre métodos contraceptivos para adolescentes: um relato de experiência. J Bras Tele., [Internet]. 2016 [cited 2019 Jan 26]; 4(2):283285. Available from: https://www.epublicacoes.uerj.br/index.php/jbtelessaude/article/view/33578/23808

14. Olympio PCAP, Alvim NAT. Board games: gerotechnology in nursing care practice. Rev Bras Enferm., [Internet]. 2018 [cited 2019 Jan 21]; 7I(suppl 2):818-26. Available from: http://www.scielo.br/pdf/reben/v7 Is2/pt_0034-7/67-reben-7|-s2-08/8.pdf

Recebido: 2020-07-27

Aceito: 2020-10-20 